\title{
Quark description of nuclear matter
}

\author{
Jürgen Berges \\ Institut für Theoretische Physik \\ Philosophenweg 16, 69120 Heidelberg, Germany
}

(HD-THEP-00-60)

\begin{abstract}
We discuss the role of an adjoint chiral condensate for color superconducting quark matter. Its presence leads to color-flavor locking in two-flavor quark matter. Color is broken completely as well as chiral symmetry in the two-flavor theory with coexisting adjoint quark-antiquark and antitriplet quark-quark condensates. The qualitative properties of this phase match the properties of ordinary nuclear matter without strange baryons. This complements earlier proposals by Schäfer and Wilczek for a quark description of hadronic phases. We show for a class of models with effective four-fermion interactions that adjoint chiral and diquark condensates do not compete, in the sense that simultaneous condensation occurs for sufficiently strong interactions in the adjoint chiral channel.
\end{abstract}




\section{Introduction}

Important progress in understanding the behavior of hadronic matter at high baryon number density has been achieved recently [1]. In QCD with three colors and three flavors of quarks the sufficiently high density phase exhibits chiral symmetry breaking and confinement which can be studied in weak coupling. The color superconducting ground state at high density is characterized by the phenomenon of color-flavor locking [2]. As a consequence all the elementary excitations carry integral electric charges and for the most part match the quantum numbers of hypernuclear matter [3, 4, 5]. At densities below the threshold for strangeness the situation seems to change dramatically: The color superconducting ground state for two quark flavors no longer breaks chiral symmetry and color symmetry is restored partially 6, 7, 8, 9, 10.

The importance of "color symmetry breaking" in the vacuum for QCD with three flavors and three colors of quarks has been recently pointed out in [11. According to [12 instanton induced interactions result in a nonvanishing color octet quark-antiquark condensate. The new vacuum exhibits colorflavor locking, confinement admits an equivalent description in a Higgs picture and the excitations match properties of the low momentum meson and baryon degrees of freedom. A consistent picture of strong interactions at long distances emerges [11].

In this letter we discuss the role of an adjoint quark-antiquark condensate at high density. We concentrate on the two-flavor limit of the octet condensate proposed in [11, 12]. This limit exhibits two-flavor color-flavor locking which leaves a global diagonal $S U(2)_{\text {color }+V}$ invariant. A more general group theoretical discussion of color-flavor locking for two quark flavors will be presented in [13]. While for three flavors condensation in the adjoint quark-antiquark channel does not change the present understanding of the high density ground state, for two quark flavors the picture changes dramatically. For simultaneous condensation in the adjoint chiral and the color-antitriplet diquark channels color symmetry is broken completely as well as chiral symmetry. The qualitative properties of this phase match the properties of nuclear matter without strange baryons, extending earlier proposals [5, 3] for a quark description of hadronic phases.

We discuss possible mechanisms for simultaneous condensation at nonzero density in effective fermionic models. We show that simultaneous condensation indeed occurs for sufficiently strong attractive interaction in the 
adjoint quark-antiquark channel. Earlier investigations studying possibilities for coexisting condensates in the standard color-singlet quark-antiquark and antitriplet diquark channels found a strong competition and no simultaneous condensation 10, 14. In this sense the adjoint chiral condensate does not compete with the diquark condensate. The result is encouraging and opens very attractive possibilities for a quark description of ordinary nuclear matter. We stress, however, that a strong attraction in the adjoint chiral channel is a necessary condition which may not be realized in the two-flavor theory and for which we cannot claim control here.

\section{The adjoint chiral condensate at high density}

Three quark flavors. In QCD with three flavors of massless quarks the Cooper pairs at high density cannot be flavor singlets, and both flavor and color symmetries are necessarily broken. A global, vector-like $S U(3)$ symmetry can be retained by the phenomenon of color-flavor locking [2]. Color-flavor locking means that left flavor rotations and transposed color rotations are performed with same opposite angles. The same happens separately with the right flavor rotations and color rotations. Since color is a vectorial symmetry this leaves a global diagonal $S U(3)_{\text {color }+V}$ and breaks chiral symmetry. In particular, all gluons acquire a mass by the Higgs mechanism.

The notion of color-flavor locking is not restricted to Cooper pairs of quarks but can also be applied to quark-antiquark condensates. This has been pointed out in [11] where a color-flavor locking quark-antiquark condensate in the octet representation is proposed in the QCD vacuum. For three massless flavors the color-flavor locking quark-antiquark $\left(\chi^{(3)}\right)$ and quark-quark $\left(\Delta^{(3)}\right)$ condensates are (omitting Dirac structure)

$$
\begin{aligned}
\chi^{(3)} & \sim\left\langle\bar{\psi}_{a}^{\alpha} \sum_{s=1}^{8}\left(\lambda_{s}\right)_{a b}\left(\lambda_{s}\right)^{\beta \alpha} \psi_{b}^{\beta}\right\rangle \\
& \equiv\left\langle\bar{\psi}_{a}^{\alpha}\left(2 \delta_{a}^{\alpha} \delta_{b}^{\beta}-\frac{2}{3} \delta_{a b} \delta^{\alpha \beta}\right) \psi_{b}^{\beta}\right\rangle, \\
\Delta^{(3)} & \sim\left\langle\psi_{a}^{\alpha} \sum_{s=1}^{8}\left(\lambda_{s}\right)_{a b}\left(\lambda_{s}\right)^{\beta \alpha} \psi_{b}^{\beta}\right\rangle
\end{aligned}
$$




$$
\begin{aligned}
& \equiv\left\langle\psi_{a}^{\alpha} \sum_{j=1}^{3}\left(\lambda_{j}^{(A)}\right)_{a b}\left(\lambda_{j}^{(A)}\right)^{\beta \alpha} \psi_{b}^{\beta}\right\rangle_{\overline{\mathbf{3}}_{\mathbf{c}}}+\left\langle\psi_{a}^{\alpha} \sum_{i=1}^{5}\left(\lambda_{i}^{(S)}\right)_{a b}\left(\lambda_{i}^{(S)}\right)^{\beta \alpha} \psi_{b}^{\beta}\right\rangle_{\mathbf{6}_{\mathbf{c}}} \\
& \equiv\left\langle\psi_{a}^{\alpha}\left(\delta_{a}^{\alpha} \delta_{b}^{\beta}-\delta_{a}^{\beta} \delta_{b}^{\alpha}\right) \psi_{b}^{\beta}\right\rangle_{\overline{\mathbf{3}}_{\mathbf{c}}}+\left\langle\psi_{a}^{\alpha}\left(\delta_{a}^{\beta} \delta_{b}^{\alpha}+\delta_{a}^{\alpha} \delta_{b}^{\beta}-\frac{2}{3} \delta_{a b} \delta^{\alpha \beta}\right) \psi_{b}^{\beta}\right\rangle_{\mathbf{6}_{\mathbf{c}}}
\end{aligned}
$$

Here $\lambda^{s}$ denote the eight Gell-Mann matrices for flavor $(a, b=1, \ldots, 8)$ and color $(\alpha, \beta=1, \ldots, 8)$. We have separated for the diquark condensate the color-antitriplet $\left(\overline{\mathbf{3}}_{\mathbf{c}}\right)$ and the color-symmetric contribution $\left(\mathbf{6}_{\mathbf{c}}\right)$. Since the antitriplet and the sextet condensate leave the same symmetries invariant any relative weight differing from one between the two contributions in (2.2) leads to color-flavor locking. The $\overline{\mathbf{3}}_{\mathbf{c}^{-}}$and the $\mathbf{6}_{\mathbf{c}^{-}}$-condensate can mix, and in general a nonzero $\overline{\mathbf{3}}_{\mathbf{c}}$-condensate induces a non-vanishing $\mathbf{6}_{\mathbf{c}}$ or vice versa. At high density the color-6 contribution to the color-flavor locked state is found to be small [2].

In the color superconducting high density phase with $\Delta^{(3)} \neq 0$ a nonzero adjoint chiral condensate $\chi^{(3)}$ does not break any new symmetriest and a nonzero $\Delta^{(3)}$ induces a non-vanishing value for $\chi^{(3)}$ [11]. At high density the color-octet condensate is, therefore, present.

Integer charges and quark description of hypernuclear matter. The color-flavor locking condensates (2.1) and (2.2) break chiral symmetry and produce gaps for all three flavors and all three colors. All gluons acquire a mass according to the Higgs phenomenon. They also break the $U(1)$ of electromagnetism but the condensates are invariant under a combination $\tilde{Q}=Q-Q_{c}$ of electric charge $Q$ and abelian color charge $Q_{c}=\frac{1}{2} \lambda_{3}+\frac{1}{2 \sqrt{3}} \lambda_{8}$ [2, 11]. Baryon number is broken only by the diquark condensate.

The dressed elementary excitations in presence of the color-flavor locking condensates carry integer electric charge with respect to $\tilde{Q}$ and for the most part match the expected features of confined hadronic matter. The gluons match the octet of vector mesons, the quark octet matches the baryon octet and an octet of collective modes due to chiral symmetry breaking is associated with the pseudoscalar octet [3, 11]. At high densities, where

\footnotetext{
${ }^{1}$ In a theory with $U(1)_{V} \times U(1)_{A}$ symmetry the diquark condensate leaves a residual $\mathbb{Z}_{2}^{L} \times \mathbb{Z}_{2}^{R}$ symmetry invariant [2]. If this were a valid symmetry the adjoint chiral condensate would vanish. Instanton-induced interactions break the $\mathbb{Z}_{2}^{L}$ and in presence of the diquark condensate and the 't Hooft vertex one can have an adjoint chiral condensate.
} 
diquark condensation breaks baryon number the quark matter description matches superfluid hypernuclear matter at densities where strange baryons are present [3, 4, 5]. Since there is no difference in symmetries between the quark and the hypernuclear matter phase there is no need for a phase transition. This is continuity as pointed out by Schäfer and Wilczek [3].

Two quark flavors. Color-flavor locking in the limit of two quark flavors can be more involved since the number of flavor and color generators do not match as in the three-flavor case. In contrast to the latter, there is in particular the possibility to form a flavor singlet diquark in the twoflavor theory which does not break chiral symmetry [6, 7, 8, 9]. Color-flavor locking may therefore not be expected in a two-flavor theory with diquark condensates only.

The two-flavor case can be obtained straightforwardly from the threeflavor case by sending the strange quark mass to infinity. In ref. [4, 5] the strange quark mass dependence of the color-flavor locking diquark state has been discussed in a theory with two massless quarks and one strange quark. For non-zero but sufficiently small strange quark mass one finds that diquark condensation leaves a global $S U(2)_{\text {color }+V}$ subgroup invariant. This is achieved by locking the left and right $S U(2)$-flavor symmetries to an $S U(2)$ subgroup of color. If the density dependent effective strange quark mass $M_{s}(\mu)$ exceeds $\simeq\left(2 \mu \Delta_{u s}\right)^{1 / 2}$, where $\Delta_{u s}$ denotes condensates which pair a strange quark with either an up or a down quark, then an unlocking transition is observed [4]. For two quark flavors, in the limit of infinite strange quark mass, no color-flavor locking and consequently no chiral symmetry breaking is observed in the diquark sector.

In refs. 10, 14 it has been shown that the standard color-singlet chiral condensate competes with the diquark condensate. No simultaneous condensation was observed and apart from the small explicit breaking due to nonzero quark masses there is no chiral symmetry breaking.

The two-flavor theory has so far not been discussed in the light of a possible adjoint chiral condensate. In ref. [13] a general group theoretical discussion of two-flavor color-flavor locking is presented. Here we restrict ourselves to consider the two-flavor (infinite strange quark mass) limit of the condensates (2.1) and (2.2) which read

$$
\chi^{(2)} \sim\left\langle\bar{\psi}_{a}^{\alpha} \sum_{s=1}^{3}\left(\tau_{s}\right)_{a b}\left(\lambda_{s}\right)^{\beta \alpha} \psi_{b}^{\beta}\right\rangle
$$




$$
\begin{aligned}
& \equiv\left\langle\bar{\psi}_{a}^{\alpha}\left(2 \delta_{a}^{\alpha} \delta_{b}^{\beta}-\delta_{a b} \delta^{\alpha \beta}\right) \psi_{b}^{\beta}\right\rangle \quad \text { for } \alpha, \beta=1,2 \\
\Delta^{(2)} & \sim\left\langle\psi_{a}^{\alpha} \sum_{s=1}^{3}\left(\tau_{s}\right)_{a b}\left(\lambda_{s}\right)^{\beta \alpha} \psi_{b}^{\beta}\right\rangle \\
& \equiv\left\langle\psi_{a}^{\alpha}\left(\tau_{2}\right)_{a b}\left(\lambda_{2}\right)^{\beta \alpha} \psi_{b}^{\beta}\right\rangle_{\overline{\mathbf{3}}_{\mathbf{c}}}+\left\langle\psi_{a}^{\alpha} \sum_{i=1}^{2}\left(\tau_{i}^{(S)}\right)_{a b}\left(\lambda_{i}^{(S)}\right)^{\beta \alpha} \psi_{b}^{\beta}\right\rangle_{\mathbf{6}_{\mathbf{c}}} \\
& \equiv\left\langle\psi_{a}^{\alpha}\left(\delta_{a}^{\alpha} \delta_{b}^{\beta}-\delta_{a}^{\beta} \delta_{b}^{\alpha}\right) \psi_{b}^{\beta}\right\rangle_{\overline{\mathbf{3}}_{\mathbf{c}}}+\left\langle\psi_{a}^{\alpha}\left(\delta_{a}^{\beta} \delta_{b}^{\alpha}+\delta_{a}^{\alpha} \delta_{b}^{\beta}-\delta_{a b} \delta^{\alpha \beta}\right) \psi_{b}^{\beta}\right\rangle_{\mathbf{6}_{\mathbf{c}}} \\
& \sim \Delta_{\overline{\mathbf{3}}_{\mathbf{c}}}^{(2)}+\Delta_{\mathbf{6}_{\mathbf{c}}}^{(2)}
\end{aligned}
$$

where the color indices in the last equation are $\alpha, \beta=1,2$. Here $\tau_{i}^{(S)}$ are the symmetric Pauli matrices and $\lambda_{i}^{(S)}$ the corresponding symmetric Gell-Mann matrices $\lambda_{1}$ and $\lambda_{3}$.

Since the adjoint quark-antiquark condensate $\chi^{(2)}$ given in (2.3) is a flavor triplet it breaks both color and flavor symmetries separately. It is invariant under the global diagonal $S U(2)_{\text {color }+V}$ subgroup which applies color and flavor transformations simultaneously. The condensate therefore locks flavor$S U(2)$ rotations to an $S U(2)$ subgroup of color and extends the notion of color-flavor locking to a pure two-flavor theory. Condensation of the form (2.3) leaves a local $U(1)_{8}$ subgroup of color unbroken. It therefore gives a mass to seven of the eight gluons by the Higgs mechanism [13].

There is a crucial difference between the two- and the three-flavor case for the diquark channel as mentioned above. The color-antitriplet part of the two-flavor diquark condensate $\Delta_{\overline{3}_{\mathrm{c}}}^{(2)}$ given in (2.4) is a flavor singlet and it doesn't break chiral symmetry. It also leaves an $S U(2)$ subgroup of color- $S U(3)$ unbroken and consequently five massless gluons remain. Therefore, for two flavors there is no color-flavor locking in the $\overline{\mathbf{3}}_{\mathbf{c}}$-channel. In contrast, a flavor-symmetric part of the condensate $\left(\Delta_{\mathbf{6}_{\mathrm{c}}}^{(2)}\right)$ can exhibit color-flavor locking, and thus breaks chiral symmetry. This possibility has been investigated in [《] where the sextet contribution was observed to vanish for sufficiently high strange quark mass. One-gluon exchange interactions relevant at asymptotically high densities do not support the $\mathbf{6}_{\mathbf{c}}$ part of (2.4). The same can be observed using effective instanton induced interactions. In contrast to the three-flavor theory the $\mathbf{6}_{\mathbf{c}}$-condensate is not induced by the color-antitriplet condensate since they leave different symmetries invariant. 
There are no indications that the two-flavor (infinite strange quark) limit of (2.2) acquires a sextet contribution and one is led to conclude that no color-flavor locking, and consequently no chiral symmetry breaking, occurs in a two-flavor theory with diquark condensates only?.

Integer charges and quark description of nuclear matter. Ordinary electromagnetism is broken in presence of the diquark condensate $\Delta^{(2)}$ or the adjoint chiral condensate $\chi^{(2)}$ but, as in the three flavor case, the condensates are invariant under the combination $\tilde{Q}=Q-\frac{1}{2} \lambda_{3}-\frac{1}{2 \sqrt{3}} \lambda_{8}$ of electric charge $Q$ and abelian color charge. In particular, all dressed excitations acquire integer charges. Baryon number is broken in presence of a diquark condensate but the color-antitriplet diquark condensate $\Delta_{\overline{\mathbf{3}}_{\mathbf{c}}}^{(2)}$ is invariant under a modified baryon number $\tilde{B}=B-\frac{1}{\sqrt{3}} \lambda_{8}$ [8].

Neither the adjoint chiral condensate nor the antitriplet diquark condensate alone break color completely. In particular, chiral symmetry is unbroken in presence of the antitriplet diquark condensate alone. It is striking to observe that in a phase with simultaneous condensation in both channels color is broken completely, all gluons acquire a gap, and chiral symmetry is broken (see also [13]). In this case the integer charged degrees of freedom for the most part match the expected features of ordinary nuclear matter! This complements the proposal set forward in [3, 5] of a quark description of nuclear matter phases. There are only two degrees of freedom, corresponding to the quarks of the third color, which carry baryon number $\tilde{B}=1$. The dressed up quark of the third color carries electric charge $\tilde{Q}=+1$ and the corresponding down quark is electrically neutral. These are the only low energy baryonic degrees of freedom and share the properties of the proton and the neutron, respectively. The remaining dressed triplet and singlet acquire a large gap and are heavy, since only the quarks of the first two colors are involved in the diquark gap. They carry integer charges $\tilde{Q}=1,0,-1$ for the triplet and $\tilde{Q}=0$ for the singlet and zero baryon number $\tilde{B}$. There is also a triplet of collective modes associated with chiral symmetry breaking. The broken symmetry generators are given by the axial charges and the massless bosons match the quantum numbers of the three pions.

\footnotetext{
${ }^{2}$ The quarks of the third color not involved in $\Delta_{\overline{\mathbf{3}}_{\mathrm{c}}}^{(2)}$ can form a condensate. In ref. [8] a small nonzero gap from quarks of the third color was found to be a few orders of magnitude smaller than the antitriplet gap using effective instanton interactions. This gap can not break the residual gauge symmetry since it only involves quarks of the third color.
} 
There is an octet of massive gluons with quantum numbers of the octet of vector mesons. It is surprising at first sight that in a pure two-flavor theory there seem to be states known from a three-flavor theory, which is a consequence of the complete breaking of color. We note that the diquark condensate contributes to the mass of the $K^{*}$ - and $\omega$-meson but not to the mass of the $\rho$-meson. The situation is similar to the fermionic sector discussed above where an additional heavy triplet and singlet appeared. The low energy theory should contain the expected two-flavor degrees of freedom. We also note that the vector meson $\sim \lambda_{8}$, which is a singlet under isospin, plays the role of the $\omega$ in the two flavor theory.

We emphasize that there is no guarantee that condensation occurs in the adjoint quark-antiquark channel. Only for sufficiently strong coupling condensation can occur in this channel. Ref. [12 points out that in threeflavor QCD the instanton induced axial anomaly results in a cubic term in the effective potential for scalar color-singlet and octet quark-antiquark states. This induces a nonzero color-octet and singlet quark-antiquark condensate in the vacuum [12]. A similar mechanism for two flavors is discussed in 15.

In the following we will show in a simple quark model that for sufficiently strong attractive interaction in the adjoint quark-antiquark channel simultaneous condensation occurs for not too high densities. The main outcome is described in fig. 2. The result is encouraging. The equivalent calculation looking for condensation in the standard chiral condensate and the color-antitriplet diquark channel found no simultaneous condensation [10, 14.

\section{A simple model for two-flavor color-flavor locking}

Color-octet and antitriplet channels. We consider a class of fermionic models similar to the Nambu-Jona-Lasinio model [17] for QCD where quarks interact via effective four-fermion interactions. The model reflects the two-flavor chiral symmetry of QCD as an $O(4) \sim S U(2)_{L} \times$ $S U(2)_{R}$ symmetry. Color is realized as a global symmetry. We will only

\footnotetext{
${ }^{3}$ In contrast, for standard mean field treatments as in 16] the instanton interaction is attractive in the Hartree approximation. The Fock contribution gives a large cancellation and renders the interaction repulsive. One-gluon exchange induced interactions, relevant at asymptotically high densities, are repulsive in the adjoint quark-antiquark channel.
} 
use this model here to describe the qualitative behavior of quarks in the high density phase and do not apply it to the vacuum. In the presence of a nonvanishing chemical potential $\mu$ for (net) quark number, the quadratic part $S_{0}$ of the Euclidean action $S=S_{0}+S_{\text {INT }}$ in momentum space ist

$$
S_{0}=\int \frac{d^{4} q}{(2 \pi)^{4}} \bar{\psi}_{a}^{\alpha}(q)\left(\gamma^{\nu} q_{\nu}+i m+i \gamma^{0} \mu\right) \psi_{a}^{\alpha}(q)
$$

with an average current quark mass $m$. The interaction part is taken to contain an interaction in the color-antitriplet diquark channel and in the color-octet quark-antiquark channel, $S_{\mathrm{INT}}=S_{\mathrm{INT}}^{(\overline{\mathbf{3}})}+S_{\mathrm{INT}}^{(\mathbf{8})}$ with

$$
\begin{aligned}
S_{\mathrm{INT}}^{\left(\overline{\mathbf{3}}_{c}\right)}= & -G_{\Delta} \int \frac{d^{4} p}{(2 \pi)^{4}}\left\{O_{(\Delta)}^{* j}[\bar{\psi} ; p] O_{(\Delta)}^{j}[\psi ; p]\right\}, \\
S_{\mathrm{INT}}^{\left(\mathbf{8}_{\mathbf{c}}\right)}= & -G_{\chi} \int \frac{d^{4} p}{(2 \pi)^{4}}\left\{O_{\left(\delta_{8}\right)}^{s i}[\psi, \bar{\psi} ;-p] O_{\left(\delta_{8}\right)}^{s i}[\psi, \bar{\psi} ; p]\right. \\
& \left.+O_{\left(\eta_{8}\right)}^{i}[\psi, \bar{\psi} ;-p] O_{\left(\eta_{8}\right)}^{i}[\psi, \bar{\psi} ; p]\right\} .
\end{aligned}
$$

Here we have defined the fermion bilinear $O_{(\Delta)}^{j}(j=1,2,3)$ carrying the quantum numbers of a Lorentz-scalar color-antitriplet diquark, the scalar color-octet flavor-triplet bilinear $O_{\left(\delta_{8}\right)}^{s i}(s=1,2,3 ; i=1, \ldots, 8)$ and the corresponding pseudoscalar flavor-singlet quark-antiquark bilinear $O_{\left(\eta_{8}\right)}^{i}$, respectively,

$$
\begin{aligned}
O_{(\Delta)}^{j} & =-\left(\psi^{T}\right)_{a}^{\alpha} C \gamma_{5}\left(\tau_{2}\right)_{a b}\left(\lambda_{j}^{(A)}\right)^{\beta \alpha} \psi_{b}^{\beta}, \\
O_{\left(\delta_{8}\right)}^{s i} & =-i \bar{\psi}_{a}^{\alpha}\left(\tau_{s}\right)_{a b}\left(\lambda_{i}\right)^{\beta \alpha} \psi_{b}^{\beta}, \\
O_{\left(\eta_{8}\right)}^{i} & =-\bar{\psi}_{a}^{\alpha} \gamma_{5}\left(\lambda_{i}\right)^{\beta \alpha} \psi_{b}^{\beta} .
\end{aligned}
$$

We will discuss a mean field solution for the low free-energy modes of the model and study condensation in the adjoint chiral channel (2.3) and the color-antitriplet diquark channel of (2.4) density with quark chemical potentials $\mu \gtrsim 350 \mathrm{MeV}$ as a reasonable lower bound motivated by the expected onset of nonzero density around $\mu \simeq m_{\text {Nucleon }} / 3$. We regulate the model by an effective cutoff scale of about

\footnotetext{
${ }^{4}$ We use the conventions employed in ref. [10].

${ }^{5}$ We will not discuss possible condensation in the pseudoscalar channel.
} 
$1 / 2-1 \mathrm{GeV}$. For the present investigation the results are rather insensitive to the details and to be explicit we implement a cutoff in three-momentum space of $\Lambda=0.7 \mathrm{GeV}$. We fix the coupling in the diquark channel, $G_{\Delta}$, to obtain color superconducting gaps of typical QCD scales of $\mathcal{O}(100 \mathrm{MeV})$ consistent with estimates in effective models and perturbative estimates [1]. (We use here a diquark gap $\Delta=125 \mathrm{MeV}$ at $\mu=500 \mathrm{MeV}$ for $G_{\chi}=0$.) We will study the phase structure of the model as a function of quark number chemical potential $\mu\left(\equiv \mu_{\text {Baryon }} / 3\right)$ and the coupling in the octet channel, $G_{\chi}$.

Based on earlier studies in [10] we expect the standard chiral condensate to be small in presence of a nonzero diquark condensate. Though a strong competition between chiral and diquark condensate was observed we note that a small chiral condensate will be present in a phase with simultaneous adjoint quark-antiquark and quark-quark condensates. This is due to the fact that in the latter phase no new symmetries are broken by a nonvanishing chiral condensate. We verified this explicitly by adding an attractive interaction to the model in the color-singlet quark-antiquark channel. The condensate turned out to be very small for sufficiently high density in the presence of simultaneous adjoint chiral and diquark condensates which we will discuss below, and we neglect the standard chiral condensate in the following.

Thermodynamic potential. The mean field effective potential $\Omega(\chi, \Delta ; \mu)$ as a function of the adjoint chiral gap parameter $\chi$ and the diquark gap parameter $\Delta$ and quark chemical potential $\mu$ can be obtained along the lines of ref. [10]. At its extrema, the potential is related to the energy density $\epsilon$, the standard quark number density $n$ and the pressure $P$ by

$$
\Omega\left(\chi_{0}, \Delta_{0} ; \mu\right)=\epsilon-\mu n=-P,
$$

where the extremum condition

$$
\left.\frac{\partial \Omega}{\partial \chi}\right|_{\chi=\chi_{0} ; \Delta=\Delta_{0}}=\left.\frac{\partial \Omega}{\partial \Delta}\right|_{\chi=\chi_{0} ; \Delta=\Delta_{0}}=0
$$

leads to coupled gap equations for $\chi_{0}$ and $\Delta_{0}$. They are related to the adjoint chiral condensate (2.3) and the condensate of Cooper pairs (2.4) by $(a, b, \alpha, \beta=1,2)$

$$
\chi_{0}=2 G_{\chi}\left\langle\bar{\psi}_{a}^{\alpha}\left(2 \delta_{a}^{\alpha} \delta_{b}^{\beta}-\delta_{a b} \delta^{\alpha \beta}\right) \psi_{b}^{\beta}\right\rangle,
$$




$$
\Delta_{0}=2 G_{\Delta}\left\langle\psi_{a}^{\alpha}\left(\delta_{a}^{\alpha} \delta_{b}^{\beta}-\delta_{a}^{\beta} \delta_{b}^{\alpha}\right) \psi_{b}^{\beta}\right\rangle
$$

The effective potential reads

$$
\begin{aligned}
& \Omega(\chi, \Delta ; \mu)=\frac{3}{4 G_{\chi}} \chi^{2}+\frac{1}{4 G_{\Delta}} \Delta^{2} \\
&-\int_{0}^{\Lambda} \frac{q^{2} d q}{2 \pi^{2}}\left\{3\left(E_{\chi}^{-}-\mu\right) \sqrt{1+\Delta^{2} /\left(E_{\chi}^{-}-\mu\right)^{2}}\left(\Theta\left(E_{\chi}^{-}-\mu\right)-\Theta\left(\mu-E_{\chi}^{-}\right)\right)\right. \\
&+ 3\left(E_{\chi}^{-}+\mu\right) \sqrt{1+\Delta^{2} /\left(E_{\chi}^{-}+\mu\right)^{2}} \\
&+\left(E_{\chi}^{+}-\mu\right) \sqrt{1+\Delta^{2} /\left(E_{\chi}^{+}-\mu\right)^{2}}\left(\Theta\left(E_{\chi}^{+}-\mu\right)-\Theta\left(\mu-E_{\chi}^{+}\right)\right) \\
&+\left(E_{\chi}^{+}+\mu\right) \sqrt{1+\Delta^{2} /\left(E_{\chi}^{+}+\mu\right)^{2}} \\
&+4(E \Theta(E-\mu)+\mu \Theta(\mu-E))+\text { constant }\}
\end{aligned}
$$

with $q=|\vec{q}|$, a field-independent $E(q)=\sqrt{q^{2}+m^{2}}$ and

$$
E_{\chi}^{-}(q)=\sqrt{q^{2}+(m-\chi)^{2}} \quad, \quad E_{\chi}^{+}(q)=\sqrt{q^{2}+(m+3 \chi)^{2}} .
$$

The constant in (3.8) does not depend on $\mu$ and is chosen such that the pressure of the physical vacuum is zero.

As $\mu$ or $G_{\chi}$ changes the potential can have several local minima in the $(\chi, \Delta)$-plane. The lowest minimum describes the lowest free energy state and is favored. Apart from the trivial vacuum with no condensation, in principle one can find three types of global minima. One corresponds to a ground state characterized by a nonzero diquark gap $\Delta \neq 0$ with $\chi=0$. This is the two-flavor color superconducting state (2SC) [6, 7, 8, 9, 10]. The other possibility is a minimum with no diquark condensate but a nonzero mass gap in the adjoint quark-antiquark channel. The third possibility is a ground state characterized by a simultaneous diquark and adjoint chiral condensate. This state exhibits two-flavor color-flavor locking (2CFL) where both chiral and color symmetries are broken completely. Fig. 11 shows an example where the potential has two degenerate minima corresponding to a first-order phase transition at which two phases have equal pressure and can coexist $\left(\mu=475 \mathrm{MeV}, G_{\chi} / G_{\Delta}=2.78, m=0\right)$ | . One minimum occurs at

\footnotetext{
${ }^{6}$ The results discussed in this work are rather insensitive to a nonzero small quark mass $m$.
} 


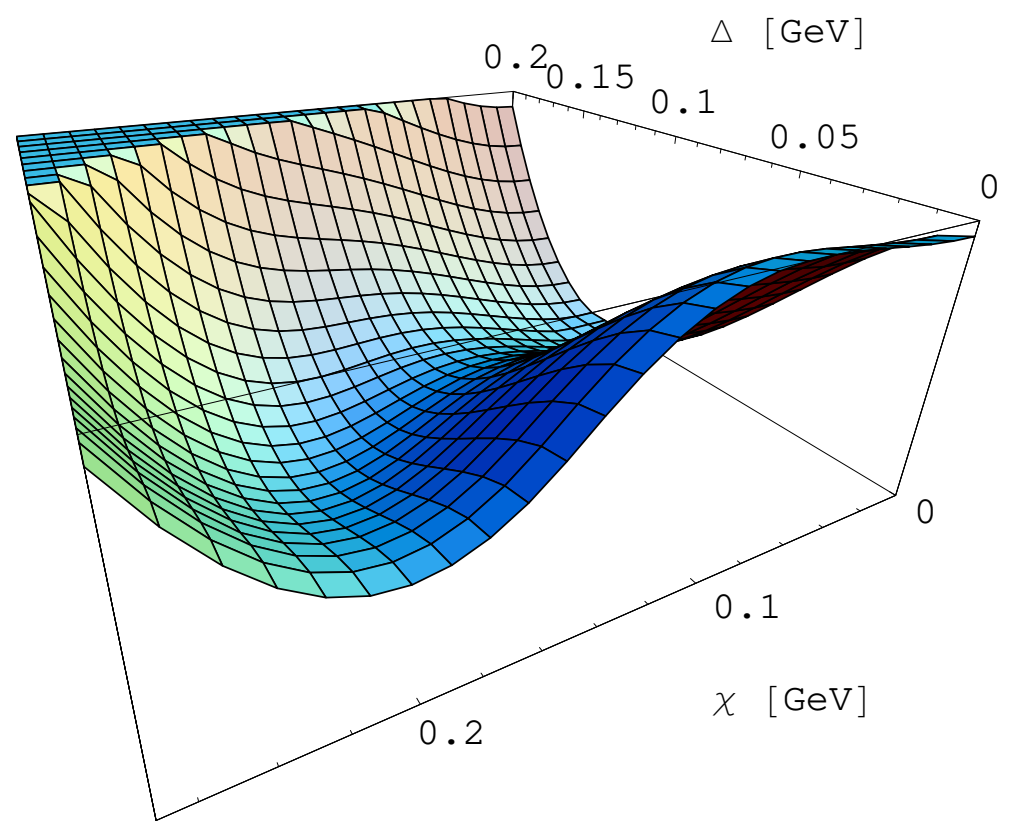

Figure 1: Effective potential at a density where 2CFL and 2SC phases coexist.

$\chi=187 \mathrm{MeV}, \Delta=88 \mathrm{MeV}$ and corresponds to the 2CFL phase. The other minimum occurs at $\chi=0, \Delta=120 \mathrm{MeV}$ and corresponds to the $2 \mathrm{SC}$ phase. For smaller $\mu$ the color-flavor locked state becomes the global minimum. We will show in the following that, for sufficiently large coupling in the adjoint chiral channel, the generic picture which emerges from the model is that a $2 \mathrm{CFL}$ phase appears once $\mu$ is lowered below a critical value.

\section{Simultaneous condensation at high density}

Two-flavor color-flavor locking. To observe a few general properties of the phase structure we consider first the possibility of a state with no diquark condensate, $\Delta=0$, and $\chi \neq 0$. At sufficiently high density or chemical potential $\mu$ such a state can not occur since more and more low momentum contributions, with momenta $q$ for which $E_{\chi}^{ \pm}(q)<\mu$, are absent in the gap equation for $\chi$ as $\mu$ increases (cf. eqs. (3.8), (3.5)). As a 


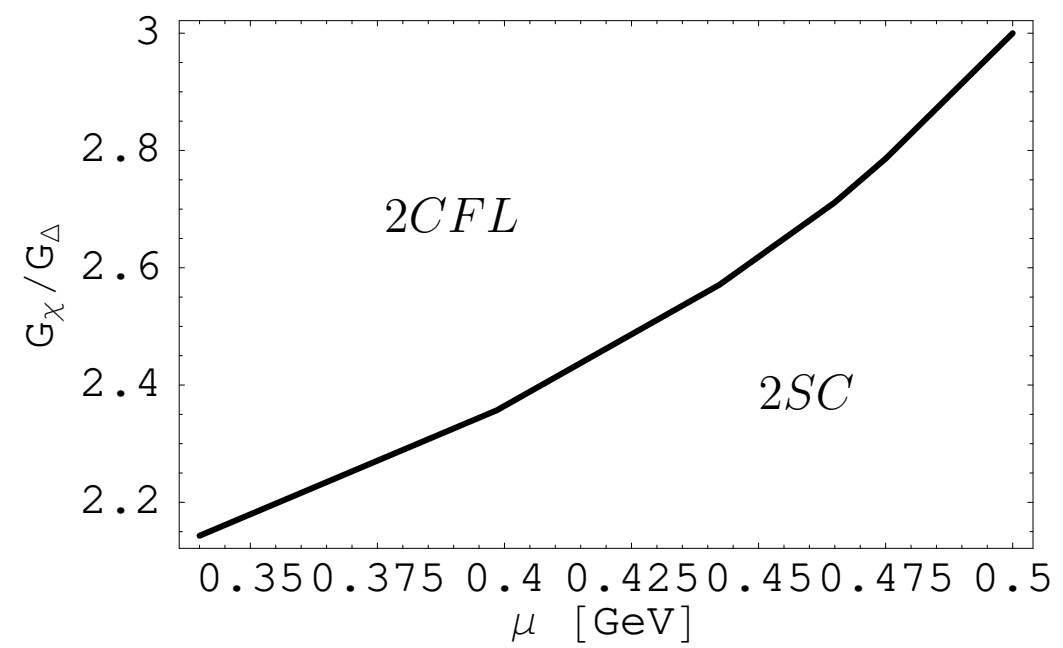

Figure 2: Phase structure as a function of quark chemical potential $\mu$ and couplings $G_{\chi} / G_{\Delta}$. Shown is the coexistence line between the 2CFL and the 2SC phase.

consequence $\chi$ vanishes for large enough $\mu$. In contrast, the BCS mechanism is operative for quark-quark Cooper pairs and ensures a nonzero diquark gap even at asymptotically high density.

A state with $\chi \neq 0$, either with zero or nonzero diquark gap, can occur at not too high densities. In fig. 2 we plot the phase diagram of the model (3.8) as a function of $\mu$ and $G_{\chi} / G_{\Delta}$. We cover a range for the chemical potential from $\mu \gtrsim 350 \mathrm{MeV}$ to $\mu \lesssim 500 \mathrm{MeV}$. For even higher values of $\mu$ the two-flavor discussion should become unappropriate since before one may expect strange quarks to be present. We observe that for a sufficiently strong coupling in the adjoint quark-antiquark channel simultaneous condensation happens always for small enough density or $\mu$ in this range. This is nontrivial, in particular, since simultaneous condensation in the diquark and the standard color-singlet quark-antiquark channel could not be observed at any density [10, 14. Though this opens striking possibilities for a quark description of ordinary nuclear matter as pointed out above, we emphasize that the possibility of quark-antiquark condensation is constrained by the strength of the coupling. For too small $G_{\chi}$ the $2 \mathrm{SC}$ phase is realized. 
Unlocking transition and onset of the strange quark. The phase diagram 2 shows an unlocking transition from the two-flavor color-flavor locked phase to the $2 \mathrm{SC}$ phase as $\mu$ increases. This phase transition is first order. Fig. 3 shows the behavior of $\chi$ (solid line) and $\Delta$ (dashed line) as a function of $\mu$ for the same couplings as in fig. 3.8. At a critical value of $\mu=475 \mathrm{MeV}$ one observes that the adjoint chiral condensate vanishes discontinuously while the diquark gap jumps to a higher value.

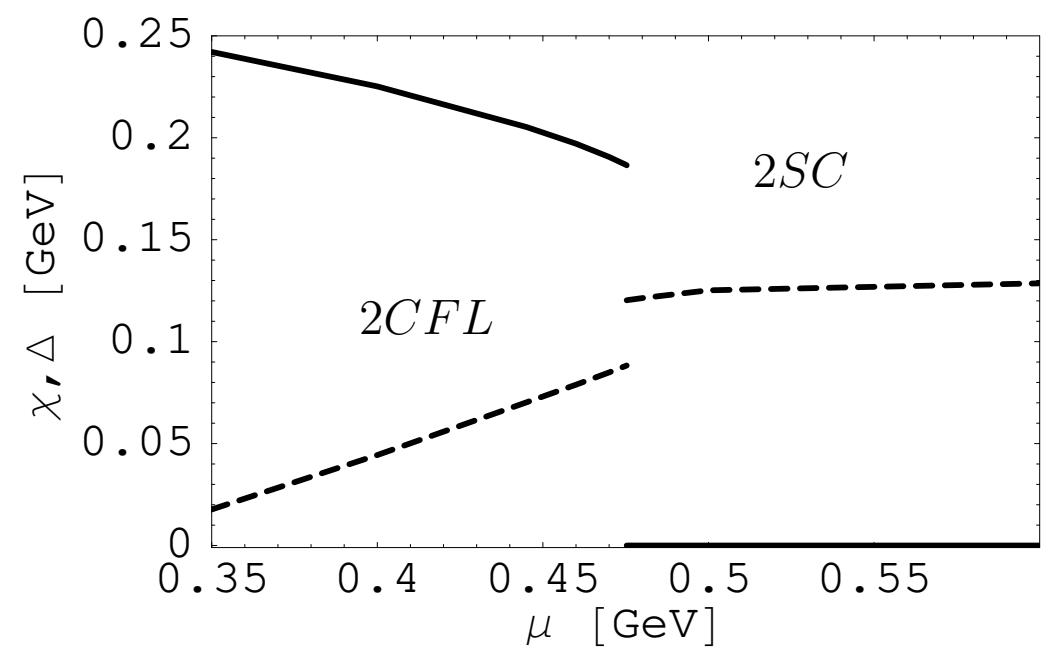

Figure 3: Adjoint quark-antiquark (solid) and diquark (dashed) gaps as a function of $\mu$ for the same couplings as in fig. 3.8. One observes an unlocking transition from the $2 \mathrm{CFL}$ phase to the $2 \mathrm{SC}$ phase at a critical $\mu$. The $2 \mathrm{SC}$ phase may not be realized if strange quarks are taken into account.

If the $2 \mathrm{CFL}$ phase is realized in nature at low enough densities then the situation as the density increases depends strongly on the value of the strange quark mass. Two qualitative different situations can occur. For sufficiently large strange quark mass the 2CFL phase would terminate in the $2 \mathrm{SC}$ phase, restoring chiral symmetry and breaking color only partially to a residual $S U(2)$. As the density is further increased one finally enters the "standard" three-flavor color-flavor locked (CFL) phase with a first order transition [4], again breaking chiral symmetry and color completely as in the 2CFL phase.

In contrast, for sufficiently small strange quark mass there can be a transition directly from the 2CFL to the three-flavor CFL phase. The additional symmetries broken during this transition are baryon number and 
strangeness. In the present model the $2 \mathrm{CFL}$ phase is no superfluid. However, if the quarks of the third color condense this would break baryon number. This is likely since the BCS mechanism guarantees a gap in presence of an arbitrarily small attractive interaction. In ref. [8] such a gap was observed in a similar model which was a few orders of magnitude smaller than the antitriplet gap. Since the quarks of the third color carry the same quantum numbers as the proton and the neutron the possible channels for condensation correspond precisely to the known possibilities for pairing in nuclear matter. This fact is valid for the 2SC phase as well and has been pointed out in [5]. We conclude that color and chiral symmetry would be broken at all nonzero densities in the latter case, with an equivalent description of confinement in the Higgs picture and qualitative properties which match the expected features of nuclear as well as hypernuclear matter.

\section{Acknowledgement}

I would like to thank Christof Wetterich and Uwe-Jens Wiese for collaboration on related work in ref. [13] and Mark Alford for helpful discussions.

\section{References}

[1] For a recent review see K. Rajagopal and F. Wilczek, hep-ph/0011333.

[2] M. Alford, K. Rajagopal and F. Wilczek, Nucl. Phys. B537 (1999) 443.

[3] T. Schäfer and F. Wilczek, Phys. Rev. Lett. 82 (1999) 3956.

[4] M. Alford, J. Berges and K. Rajagopal, Nucl. Phys. B558 (1999) 219.

[5] T. Schäfer and F. Wilczek, Phys. Rev. D60 (1999) 074014.

[6] B. Barrois, Nucl. Phys. B129 (1977) 390. S. Frautschi, Proceedings of workshop on hadronic matter at extreme density, Erice 1978.

[7] D. Bailin and A. Love, Phys. Rept. 107 (1984) 325, and references therein.

[8] M. Alford, K. Rajagopal and F. Wilczek, Phys. Lett. B422 (1998) 247. 
[9] R. Rapp, T. Schäfer, E. V. Shuryak and M. Velkovsky, Phys. Rev. Lett. 81 (1998) 53.

[10] J. Berges and K. Rajagopal, Nucl. Phys. B538 (1999) 215.

[11] C. Wetterich, hep-ph/0008150; Phys. Lett. B462 (1999) 164; hepph/9908514.

[12] C. Wetterich, hep-ph/0011076.

[13] J. Berges, U.-J. Wiese and C. Wetterich, in preparation.

[14] G. Carter and D. Diakonov, Phys. Rev. D60 (1999) 016004.

[15] J. Berges and C. Wetterich, in preparation.

[16] S.P. Klevansky, Rev. Mod. Phys. 64 (1992) 649.

[17] Y. Nambu and G. Jona-Lasinio, Phys. Rev. 122 (1961) 345; 124 (1961) 246. 